\title{
Modelling of Things on the Internet for the Search by the Human Brain
}

\author{
Fernando Luis-Ferreira $^{1,2}$ and Ricardo Jardim-Gonçalves ${ }^{1,2}$ \\ ${ }^{1}$ Departamento de Engenharia Electrotécnica, Faculdade de Ciências e Tecnologia, FCT, \\ Universidade Nova de Lisboa, 2829-516 Caparica, Portugal \\ ${ }^{2}$ Centre of Technology and Systems, CTS, UNINOVA, 2829-516 Caparica, Portugal \\ $\{f l f, r g\} @ u n i n o v a . p t$
}

\begin{abstract}
The Internet has become the main source of information for business and research activities. Despite the value of libraries supported by computational cataloging, there are far more opportunities to retrieve information on the Internet than in paper books. However, when we seek the Internet we get essentially chunks of text with titles and descriptors resulting from search engine's activity. Albeit some information may contain sensorial or emotional contents, the search results come essentially from algorithmic execution over keywords by relevance. Our brain retrieves information about things in real world by capturing sensorial information and storing it with emotional experience. We can question why things in Internet are not represented in a similar way to human brain. The present research aims to support a new type of search by sensations and emotions in a path to model Things in Internet towards a human-like representation of objects and events, based on lessons learned from the human brain.
\end{abstract}

Keywords: Knowledge Management, Neuroscience, Sensation and Perception, Internet of Things, Interoperability.

\section{Introduction}

In the last years, the Internet has evolved towards user's needs in terms of expression of ideas with a boom in the blogosphere and then with the social networks allowing a real-time expression of actions and thoughts. With a computer or a smartphone, a person can express ideas and report events putting it online in murals or tweeting events as they happen. We can talk about a freedom of speech as anyone can say what they want and publish it to be available anywhere in the world. Diverse approaches where developed for users to organize their photos, to share whatever they want with friends colleagues or to all people. We may wonder what else could be provided to users that are more than a support for text and image or sound. The research reported in this paper addresses new approaches for users to interact with the web or with each other using the web.

The main vision of the current work is to learn from the human sensorial interaction with the world and the way we perceive the world mediated with emotions. In general, 
the authors want to learn and, as much as possible, replicate how the brain and the nervous system handles information and generates knowledge and wisdom. The research presented in this paper seeks for an enlarged sensorial representation of objects in the Internet and, in some cases, mediated with emotional information. The relevance of this work is extensible from the individual representation of episodes of a person's life, as depicted in this paper (Fig. 3), to the opportunity of an enlarged object representation, either in an Internet of Things (IoT) context or, in a broad sense, as a generalization in the Internet with potential impact for Business, Social Networking or Education.

In the next section, a modification is proposed on how Things are handled in the Internet, on an IoT context, by means of an enrichment of the description of those Things that relies on the inclusion of human perceptions and emotions.

In section 3, the presented Research is contextualized in the framework of the ongoing doctoral studies. In section 4 the Methods and Associated concepts developed in the current research are presented highlighting its application in a knowledge management framework, and finally, section 5 presents Conclusions and Future work.

\section{Relationship to Internet of Things}

Internet of Things proposes an environment littered with communicating objects, with a pervasive presence around us of a variety of things, or objects, such as RadioFrequency Identification (RFID), tags, sensors, actuators, mobile phones, etc. These networked objects will play its role in both working and domestic fields, with scenarios in domotics, assisted living, e-health and enhanced learning as possibilities for this new paradigm with a leading role in a near future [1]. Considering the proposed different layers for an IoT architecture, it can be designed with a path starting on a perception layer, being its main function to identify objects and gather information. It is formed mainly by sensors and actuators, monitoring stations (such as cell phone, tablet PC, smart phone, PDA, etc.), nano-nodes, RFID tags and readers/writers [2]. But what can those assets tell us about human beings? Can they contribute to our perception of the world? Are they an extension of our sensorial organs? They can tell us about goods, flow of materials and technological devices, but what can they tell about people? From the development of cyber-physical systems (CPS) results a new range of possibilities of interaction with the physical world around us [3] but in order to advance that interaction a step ahead, we propose that computational resources meet our biological perception of the world by considering our sensorial capabilities. To close this loop, the inclusion of emotional information will better characterize objects or events by allowing an emotion-driven management of those objects. Considering the relevance of sensorial and emotional information as well as our relation with the world, by means of sensations and emotional regulation, we propose a model for representing things, supporting sensations and emotional information as depicted in the next figure. It represents a model that includes an 


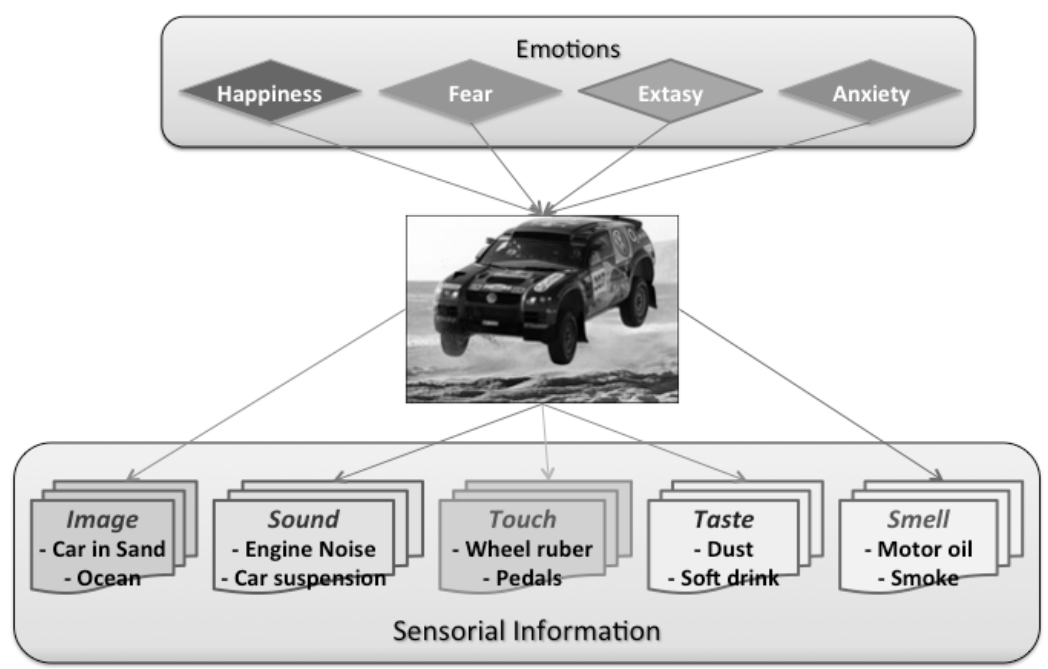

Fig. 1. Modelling things to include Sensations and Emotions

association of sensorial information, as humans perceive Things in real world, along with support for emotional response to those objects or events

The use of tagging technics, either automatic or made by users [4] will allow the description of objects, in a first stage, with either symbolic or textual information. In a later stage, as technology makes available new devices, we envisage that information can be retrieved by portable non-invasive devices. In an ideal scenario, using this model along sensorial and emotion-catching devices in an IoT world, it would be possible to automatically acquire sensorial and emotional information.

\section{Followed Research Method}

The on-going research is following the traditional research method based on research questions that result from a background observation with an hypothesis that will be tested over a design experiment, leading to results to be analysed and published on its major findings. The research path is focused on improving information systems, especially its aspects over the internet, taking examples from existing neuroscience models, in particular the Two-Stream Hypothesis [5], as a source of guidance for knowledge management for its perspective on a "where" and a "what" streams that also seem to be useful in a IoT vision of our interaction with the connected world.

\subsection{Research Question}

The research question defines the area of the interest for the authors and the problem they want to address. The selected question; 
Q - How to improve information systems based on lessons learned from neuropsychology and neurophysiology?

Is decomposed in the following sub-questions

Sq1 - Does Sensorial and Emotional Information brings an added value to Internet objects for human usage?

Sq2 - Would the two-stream Hypothesis of the brain be suitable for taking advantage of sensorial and emotional information for knowledge management?

Those questions will be addressed with analogous kind of sensorial perception and emotional contextualization in handling Internet Objects. To pursue such approach some scientific challenges will be addressed on how to model things in the Internet with sensorial and emotional information for the search by the human brain.

\subsection{Background Observation}

The Internet as an information system takes us to a world mostly expressed on textual information. As support to written data there are pictures, music and movies. The human brain uses the five senses to retrieve, store, handle and later, seek for information to think and to establish correlations with newly acquired information.

Many brain models have been constructed based on experiments and observation. From hundreds of years most of the great classical philosophers like Plato, Aristotle, Spinoza, Descartes, Hobbes and Hume, had recognizable theories of emotion [6] tried to develop cognitive models and understand how the mind works. Since those times many things changed. Today with Internet's worldwide diffusion and its vast amount of information, would be desirable to use it as a veritable extension of the human brain [7], but in order to make it possible it is logical to support, along with the growth of information, new ways of representing objects as perceived by the human brain. In our research we consider that an unavoidable method, towards a brain-like representation of objects, is to include sensorial and emotional information.

Considerations about the state of art on sensorial information in the Internet were made in our previous paper [8] where we propose a framework for knowledge management using the two-stream hypothesis. From that framework, based on sensations and emotions, it is necessary to go beyond state of art and develop models that would allow a human-like representation of Objects and Episodes. By Episodes, or i-episodes, we define the possibility of storing interconnected sensorial and emotional information segments, on i-episode, were that information makes sense as a whole to the person who experienced that place at that time. In the same line, we can relate sensorial and emotional experience of a person towards a Thing. In the next section the proposed hypothesis explore research paths to accomplish those goals.

\subsection{Hypothesis}

If we adopt selected brain models in order to improve knowledge management in particular or in the Internet, then the probability of presenting and finding information that meets our ways of perceiving the world will be higher. Getting more concrete with the following construction; 1) A new conceptualization of knowledge 
representation can support sensorial and emotional information 2) Support for meta information can foster sensorial and emotional representation methods 3) Semantic mediation can be supported by enlarged information and thus improving knowledge extraction and search results. With this in mind we will extend the sensorial information to the five senses, or at least enable that potential, also associating emotions textually expressed or device captured. Our primary goal is to facilitate better knowledge management by enabling different types of information representation and extraction based in sensorial and emotional tagging.

\section{Methods and Associated Concepts}

In our first exploration of the question, we took a look at a brain model and explored how it could be valuable to be applied in a technological framework. The basis for that was to observe that we receive images that are formed in the occipital region of the brain [9], then according to the two-stream hypothesis, the dorsal stream goes from the occipital lobe to the temporal lobe and is known as the "what stream" and the Ventral Stream goes from the occipital lobe to the temporal lobe and is known as the "where stream"[5].

Two findings are relevant in this theory; first, the brain tries to identify what is in the visual field, identifying Objects, mostly by comparing with memories. Then the brain tries to give spatial context by establishing a sense of location for the visual information Episodes. So the cognitive process starts with images at the visual cortex, then two streams follow a path thru other regions of the brain where the same process of analysis and comparison to existing records is extended to other recorded sensorial information, thus empowering the what and where context findings. And that is the area where, from our research strategy, technology meets the brain. The proposed approach relies on the establishment of a databank of sensations that could help us identify "what" are the Things in real world as we capture sensations. Then it can result in Episodes, if for a given person, a relation is established between a set of sensations giving us the "where". As in the brain, it is supposed to store emotions associated with those sensations that give relevance to the objects or to events.

\section{Handling Emotions in Internet}

Emotions are what make us humans. They give meaning to our existence [10]. They reflect the effect caused on humans by the sensorial experience of the world. An important part of our information gathering behavior has always been to find out what other people think [11], thoughts and our perception of the world result in an emotional response that can be consciously assimilated and can simultaneously produce a physiological response. In affective computing, we can separately examine functions that are not so easily separated in humans [12]. We identified a lack of applications that consider emotion related aspects. This situation is mainly due to the great amount of proposed theoretical models and the complexity of human emotions [13]. So, in order to proceed with our framework design, we need to include emotions along with sensorial information, both represented, in an early stage, with tags. 


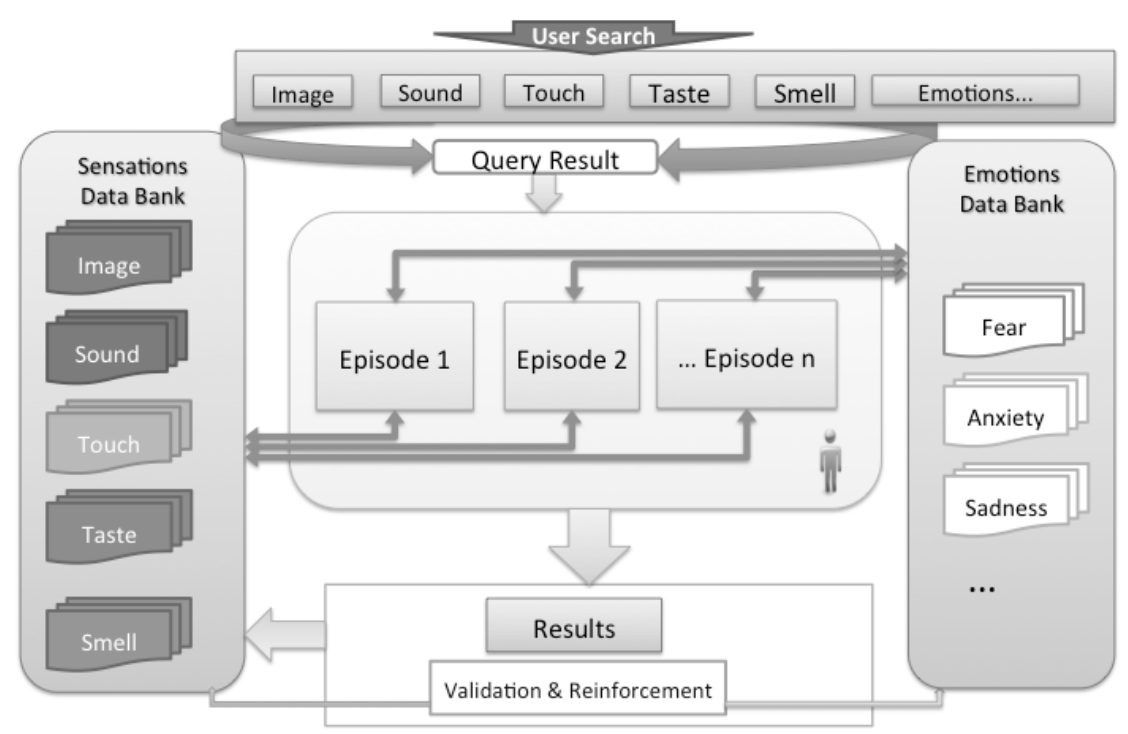

Fig. 2. Proposed Framework for Knowledge Management

This framework proposes an enlarged search using emotions and sensations that will result in episodes, being those unitary or complex and from a person or others. This search will be done in specific databanks with the most diverse sensations and emotions that, at the same time, consider clusters named Episodes. When an episode is validated by one or more users it will be reinforced and progress in relevance.

The next challenge to the ongoing research is to gather and store related pieces of sensorial and emotional information in the form of episodes, a so called i-Episode, that makes sense to a person or to a group of persons. The result of that new type of search may be validated by the user and reinforce a possible classification e.g. beautiful sunset, amazing jump, good scent. The stored information, if publicly available, can be used by others to represent their sensations or reproduce their events.

\subsection{Design Experiment}

We want to prove that a framework inspired in the two-stream hypothesis, as described above, has a great potential for handling information in innovative and fertile knowledge bases. In order to accomplish that goal we need a new modelling of Things, similar to what our brain does, which is also in the scope of the research reported in this paper. In order to accomplish that goal, we exploit the retrieval and storage of information as it happens based on the two-stream hypothesis. This can be achieved by establishing a connection with 'where' it was and 'what' is represented. Then it will instantiate a knowledge base and harmonise it with existing information, which includes sensorial and emotional information, either by properly instantiate with retrieved knowledge or by semantic annotation. This could be backed by semantically-based Web Map Mediation Services a core of semantic and ontological 
tools for mapping [14], mediation, annotation and what else found needed for pursuing the most consensual and interoperable solution as possible.

In the next picture the proposed modelling approach supported by both a databank of emotions and other for sensations, with correlations that can be associated as episodes of a person's experience in life. Those data banks can be populated by known sensations and emotions and will permanently be updated with user's contributions that can be used by them or by others. When performing a query it is possible to find our own episodes but also other's episodes if available publicly. The resulting queries, if validated by users can increase the bonds between objects and associated emotions or perceptions.

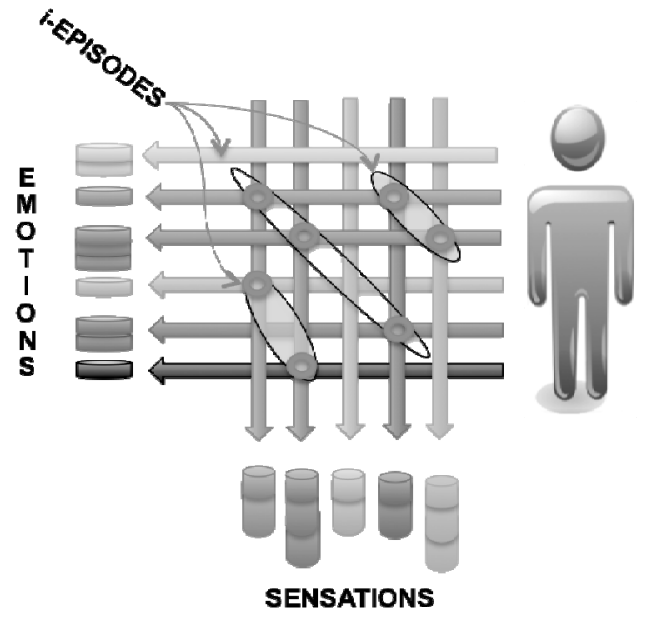

Fig. 3. Emotions and Sensations collected and clustered for a person as i-Episodes

As an example, if surfers associate a beach with good waves, soft sand and smooth sea scent then the occurrence of more validated results will reinforce such associations. In figure 3, three instantiations of the proposed data model result in a person's set of episodes. Those episodes encompass selected emotions and perceptions for the person's i-Episode description. Both emotions and perceptions are stored in specific databases, which use existing content or the user's description, or measurements. As a result, a new form of modelling information allows richer sensorial descriptions and empowers existing knowledge. In terms of functionality, new services can be deployed, making use of sensorial and emotional information, providing more ubiquitously searching and finding of information. Comparing to existing solutions, we propose a framework that supports; 1) a new data model including sensorial an emotional information along with 'traditional' data. 2) a new approach to search on the internet by allowing new specific fields with the proposed data model 3) a modelling approach that supports correlations between sensations and emotions. As a result we want to change the established paradigm of Internet object description, allowing new methods for knowledge management. The first stage consists in presenting a new data model that support sensorial and emotional information starting its filling process with an annotation sequence that is supported by an ontology [15]. On a second stage a framework is being developed to support the new object modelling, its storage, usage and retrieval in a seamless and standardized operating mode. Finally the objective will be to establish a methodology for the association process of sensorial and emotional information in order to promote the existence of i-Episodes. The final result will be a permanently growing knowledge base of Emotions, Sensations and Episodes. As an instantiation of what is described, imagine that a person stores diverse Episodes in life. These episodes can include sport 
events, music concerts and other events where the person describes sensations and emotions. With this reach variety of information it is possible that other persons attending the same events will able to find them on the Internet even with some disability, e.g. a blind can find the environment of a rock concert by searching the music and the food served at that precise event. People can share memories using sensations and emotions in databank, building their own set of Episodes.

\section{$5 \quad$ Concluding Remarks and Future Work}

The present work describes an approach to incorporate sensations and emotions in information available on the Internet but also to establish relations between different objects and its sensorial and emotional relation to persons. This is a challenging question and addresses a person's centric content for the Internet. To accomplish those objectives we want to enable the creation of a person's own episodes that are more than pictures, by the inclusion of their extended sensorial and emotional experience. At the present stage the authors to pursue the establishment of a bank of emotions and sensations that could be accessed by the person or by others when possible. The case applies for people sharing the same type of event, e.g. people attending the same party, the same sports event or even the same natural phenomena. Our objective is gaining consistency as we are enlarging the possibility of representing emotions and sensations, starting with the usage of tags, but allowing other representations of captured information from current, emerging and future technologies. In an ideal situation those episodes would be captured by portable or wearable devices or by communicating with the surrounding IoT connected devices.

The novelty of the present work can be identified by the possibility of retrieve information related with the five senses and user' emotions as part of Object's representation and use it to increase the description of those Things. Then we explore the possibility of interconnecting that information as Episodes as in fact happens with a person's experience. The conceptual goal addresses lessons learned from the TwoStream Hypothesis trying to find what is searched and where thus it exist or where thus that happened. Future work for the proposed framework will be to support the establishment of relations envisaging the permanence of Events on the Internet. This research may impact in many societal and business aspects. Just to name same, people with sensorial limitation, either blind or deaf, will have more chances to explore the Internet and have a more immersive experience with the expanded emotional and sensorial information. In business, enhanced product description will increase chances to reach a wider range of potential customers and better description of products, from raw material to consumer goods, will empower an advantage over the concurrence.

\section{References}

1. Atzori, L., Iera, A., Morabito, G.: The Internet of Things: A survey. Computer Networks 54(15), 2787-2805 (2010)

2. Domingo, M.C.: An overview of the Internet of Things for people with disabilities. Journal of Network and Computer Applications 35(2), 584-596 (2012) 
3. (Raj) Rajkumar, R., Lee, I., Sha, L., Stankovic, J.: Cyber-physical systems. In: Proceedings of the 47th Design Automation Conference - DAC 2010, p. 731 (2010)

4. Liu, D., Wang, M., Hua, X.-S., Zhang, H.-J.: Semi-Automatic Tagging of Photo Albums via Exemplar Selection and Tag Inference. IEEE Transactions on Multimedia 13(1), 82-91 (2011)

5. Mishkin, M., Ungerleider, L.G., Kathleen, A.: Object vision and spatial vision: two cortical pathways. Trends in Neuroscience 6, 414-417 (1983)

6. de Sousa, R.: Emotion. The Stanford Encyclopedia of Philosophy (2012), http://plato.stanford. edu/archives/spr2012/entries/emotion/ (accessed: November 11, 2012)

7. Maurer, H., Tochtermann, K.: On a New Powerful Model for Knowledge Management and its Applications. Journal of Universal Computer Science (2002), http: / /www . jucs . org/ jucs_8_1/on_a_new_powerful/maurer_h.html (accessed: November 11, 2012)

8. Ferreira, F., Jardim-Goncalves, R.: Framework for Knowledge Management Based in the Two-Stream Hypothesis. In: Camarinha-Matos, L.M., Shahamatnia, E., Nunes, G. (eds.) DoCEIS 2012. IFIP AICT, vol. 372, pp. 69-76. Springer, Heidelberg (2012)

9. Netter, F.H.: Atlas of Human Anatomy, 2nd edn. Novartis (1990)

10. Hilligsoe, S., Jackobsen, H.: Negotiation: The art of reaching agreement. Academica (2009)

11. Pang, B., Lee, L.: Scientific Commons: Opinion Mining and Sentiment Analysis. Foundations and Trends® in Information Retrieval 2(1-2), 1-135 (2009)

12. López, J.M., Gil, R., García, R., Cearreta, I., Garay, N.: Towards an ontology for describing emotions. In: Lytras, M.D., Damiani, E., Tennyson, R.D. (eds.) WSKS 2008. LNCS (LNAI), vol. 5288, pp. 96-104. Springer, Heidelberg (2008)

13. Pinker, S.: So How Does the Mind Work? Main 20(1), 1-24 (2005)

14. Gahegan, M., Smart, W., Masoud-Ansari, S., Whitehead, B.: A semantic web map mediation service. In: Proceedings of the 1st ACM SIGSPATIAL International Workshop on Spatial Semantics and Ontologies - SSO 2011, pp. 1-8 (2011)

15. Talantikite, H.N., Aissani, D., Boudjlida, N.: Semantic annotations for web services discovery and composition. Computer Standards \& Interfaces 31(6), 1108-1117 (2009) 